Annuaire suisse de politique de développement

\title{
Le label ZEWO attribué aux organisations d'utilité publique : une contribution pour une meilleure transparence sur le marché du don en Suisse
}

\section{Ariuscha Davatz}

\section{(2) OpenEdition}

Journals

Édition électronique

URL : http://journals.openedition.org/aspd/455

DOI : $10.4000 /$ aspd. 455

ISSN : 1663-9669

Éditeur

Institut de hautes études internationales et du développement

Édition imprimée

Date de publication : 1 novembre 2004

Pagination : 109-116

ISSN : 1660-5934

\section{Référence électronique}

Ariuscha Davatz, «Le label ZEWO attribué aux organisations d'utilité publique : une contribution pour une meilleure transparence sur le marché du don en Suisse », Annuaire suisse de politique de développement [En ligne], 23-2 | 2004, mis en ligne le 10 mars 2010, consulté le 08 septembre 2020 URL : http://journals.openedition.org/aspd/455 ; DOI : https://doi.org/10.4000/aspd.455 


\title{
Le label ZEWO attribué aux organisations d'utilité publique: une contribution pour une meilleure transparence sur le marché du don en Suisse
}

\author{
Ariuscha Davatz*
}

\section{Histoire de la ZEWO}

Dès 1934, les donateurs suisses ont voulu savoir dans quelle mesure certaines organisations d'utilité publique étaient dignes de confiance, avant de leur donner de l'argent. Un besoin auquel la Conférence nationale suisse de l'action sociale (LAKO) et la Société suisse d'utilité publique ont répondu en créant le Bureau central d'information en 1934 et l'association ZEWO (Bureau central des œuvres de bienfaisance) en 1936. Le label de qualité, destiné aux organisations d'utilité publique collectant des dons, fut quant à lui créé en 1942. Les organisations qui reçurent ce label étaient prêtes à respecter des normes supplémentaires, en plus des exigences minimales légales. Depuis sa création, la ZEWO tient un service d'information destiné aux donateurs. Par ailleurs, elle publie depuis 1946 le Calendrier suisse des collectes.

Le 20 juin 2001, l'assemblée des membres a décidé de transformer l'association en une fondation afin d'en renforcer l'indépendance par rapport aux œuvres d'entraide.

\section{La ZEWO aujourd'hui}

La fondation ZEWO a pour objectif de promouvoir vis-à-vis du public la transparence et l'intégrité des organisations d'utilité publique collectant des dons. Elle leur attribue donc un label de qualité, dont elle s'emploie à assurer la notoriété. Le label est attribué pour cinq ans : à l'échéance de ce délai, lors de la procédure de renouvellement de la certification, les organisations font à nouveau l'objet d'un examen portant sur le respect des exigences de la fondation. De son côté, celle-ci coordonne les collectes des principales œuvres d'entraide dans le Calendrier suisse des collectes. Pour les donateurs, elle gère un service d'information sur les organisations titulaires ou non du label de qualité. Elle établit en outre chaque année une statistique des dons.

Pour la création de la fondation, la Société suisse d'utilité publique a mis à disposition un capital de départ, complété par les contributions versées par 20 cantons, en signe de solidarité avec la ZEWO. Le Conseil de fondation est composé de représentants des organisations d'utilité publique et d'associations de protection des consommateurs ainsi que de représentants des milieux politique

* Service d'information et de collecte des dons, Fondation ZEWO. 
et économique et du domaine social. Actuellement, le Conseil de fondation compte trois représentants d'œuvres d'entraide parmi ses neuf membres.

Aujourd'hui en Suisse, près de 450 organisations d'utilité publique collectant des dons, elles-mêmes divisées en organisations simples, en organisations mères et en sous-organisations, sont habilitées à porter le label ZEWO. Sur quelque 240 organisations simples et organisations mères, seules 65 organisations opèrent dans le domaine de la coopération au développement.

Les critères d'attribution du label de qualité ZEWO

La ZEWO octroie la certification aux organisations d'utilité publique, c'està-dire aux œuvres d'entraide qui remplissent des missions sociales, humanitaires ou socioculturelles ou dont l'activité est consacrée à l'environnement.

Parmi les principaux critères que doit remplir une organisation pour obtenir le label ZEWO figurent des structures efficaces de contrôle interne et externe, une information claire sur les activités et l'utilisation des fonds, la transparence des comptes ainsi que l'utilisation rentable, efficace et adaptée des dons. La ZEWO vérifie, complète et adapte constamment ses critères. Nombre d'entre eux jouent également un rôle déterminant dans la corporate governance (gouvernance d'entreprise). Les principales exigences de la ZEWO et leur lien avec la corporate governance sont expliqués brièvement ci-après.

\section{Contrôle interne}

Le contrôle interne porte fondamentalement sur la répartition des tâches et sur la collaboration entre le niveau stratégique et l'exécutif. La ZEWO exige une séparation stricte de l'organe de direction et de l'organe exécutif afin que le premier puisse réaliser avec compétence ses tâches de direction et de surveillance. La ZEWO impose par ailleurs que les membres de l'organe de direction ne soient pas personnellement liés au gérant de l'organisation (liens du mariage, de parenté, de parenté par alliance, cohabitation). Cela afin de garantir un contrôle efficace de la gestion par l'organe de direction et une séparation claire des pouvoirs. Celle-ci permet par ailleurs à l'organe de direction de définir les objectifs de l'organisation et de décider des questions stratégiques. Enfin, l'organe de direction peut choisir le management et contrôler la réalisation des objectifs. Ainsi, la séparation entre l'organe de direction et l'organe exécutif permet un contrôle effectif du pouvoir.

La ZEWO attache beaucoup d'importance à ce que les décisions de l'organe de direction soient largement soutenues. Ce dernier doit donc comprendre au moins cinq membres qui ne soient pas personnellement liés les uns aux autres. Le droit de signature collective garantit en outre que le pouvoir de représentation ne soit pas détenu par une seule et même personne.

Un contrôle interne adapté doit également assurer le déroulement régulier de l'activité de l'organisation. La ZEWO exige en outre que la répartition des compétences entre l'organe de direction et l'organe exécutif soit clairement stipulée dans un règlement d'activité. Le comité directeur ou le conseil de fondation assume, en sa qualité d'organe de direction, la direction suprême et endosse la 
responsabilité quant à la réalisation de l'objectif au moyen de mesures adaptées, efficaces et rentables.

Les critères fixés par la ZEWO garantissent ainsi la séparation des pouvoirs entre l'organe de direction et l'organe exécutif, ce également en vue de la corporate governance.

\section{Travail professionnel au sein du comité directeur ou du conseil de fondation}

Outre la séparation stricte entre l'organe de direction et l'organe exécutif, il est important que les fonctions de contrôle et de surveillance puissent être assurées par un comité directeur ou un conseil de fondation suffisamment qualifiés, notamment au sein des organisations importantes, où la direction a de nombreuses tâches à remplir. Celles-ci sont en principe réalisées à titre bénévole. Certains membres qualifiés du conseil de fondation et du comité directeur demandent toutefois un dédommagement pour la réalisation de travaux complexes. C'est pourquoi la ZEWO a adapté sa disposition sur l'activité bénévole du comité directeur ou du conseil de fondation et autorise également, depuis peu, le versement d'indemnités à l'organe de direction. Malgré tout, il faut toujours tenir compte du fait qu'il s'agit d'une organisation d'utilité publique et que les membres du comité directeur ou du conseil de fondation doivent effectuer au moins 100 heures à titre bénévole. Des indemnités peuvent être versées pour les heures supplémentaires, à hauteur des exigences liées aux travaux exécutés.

\section{Contrôle de gestion}

L'organe de révision remplit une fonction de contrôle dans le cadre de la corporate governance par rapport à l'organe de direction et de gestion et veille à ce que les fonds soient utilisés à bon escient. Il vérifie si la comptabilité, le bilan et le compte d'exploitation sont conformes à la loi et aux statuts et si l'organe de direction remet des rapports d'activité en bonne et due forme.

La ZEWO exige de toutes les organisations titulaires du label ZEWO qu'elles fassent réviser leur comptabilité et leurs comptes annuels par une instance techniquement habilitée et indépendante par rapport aux organes de direction et de gestion. Plus l'organisation est importante et complexe, plus les exigences imposées à l'organe de révision en matière d'habilitation sont élevées. Le contrôle doit être effectué conformément aux règles d'organisation et d'éthique professionnelle de la Chambre fiduciaire. L'organe de révision doit confirmer dans son rapport qu'il est indépendant et qu'il n'est soumis à aucune pression coercitive. Son travail consiste à contrôler si la comptabilité et les comptes annuels sont bien conformes aux dispositions légales suisses, aux statuts et aux dispositions ZEWO en vigueur. Grâce à cette réglementation, la ZEWO s'assure que les organes de direction des organisations titulaires du label ZEWO rendent compte de façon correcte de l'utilisation des fonds.

\section{Transparence vis-à-vis du public}

La ZEWO accorde beaucoup d'importance à la transparence des institutions visà-vis de leurs groupes d'interlocuteurs et de leurs bailleurs de fonds, un sujet qui 
joue également un rôle essentiel au niveau de la corporate governance. Ainsi, les institutions d'utilité publique collectant des dons sont tenues de fournir à leurs interlocuteurs des informations fiables et appropriées sur leurs structures.

Le cadre légal ne prévoyant aucun droit d'information pour les donateurs et les sponsors, la ZEWO a inclus dans son règlement relatif à la collecte de fonds une disposition qui oblige les institutions d'utilité publique collectant des dons à fournir des renseignements et de la documentation à leurs donateurs, à leurs membres et à leurs autres interlocuteurs, si ceux-ci en font la demande. Un rapport et des comptes annuels détaillés font partie des principaux outils d'information des organisations.

\section{Intégrité et éthique}

La communication avec les donateurs et le grand public doit respecter les principes de l'éthique et de l'intégrité. Il s'agit, en particulier, de fournir des données appropriées et conformes à la réalité dans les appels de fonds et dans les autres publications utilisées dans le cadre de collectes. En effet, il est hors de question de tenter d'influer sur les dons du public en déformant ou en exagérant les faits, de manière à jouer sur l'émotionnel. De plus, la dignité des êtres humains à qui les dons sont destinés doit toujours être préservée. La ZEWO refuse par ailleurs de soutenir les parrainages individuels d'enfants et de faire de la publicité dans ce domaine, préférant privilégier le soutien à des projets globaux.

\section{Nouvelles recommandations relatives à la présentation des comptes (Swiss GAAP RPC 21)}

L'objectif des nouvelles recommandations relatives à la présentation des comptes (Swiss GAAP RPC 21) est de standardiser les comptes annuels des œuvres d'entraide, afin de faciliter les comparaisons et d'accroître la transparence. Toutes les institutions titulaires du label de qualité ZEWO ont l'obligation d'appliquer ces recommandations, qui prennent en considération des particularités telles que l'absence de but lucratif et les méthodes de recherche de fonds des organisations d'utilité publique. Les grosses structures devront se conformer à la RPC 21 dès l'exercice 2004; les plus petites ont, quant à elles, une année de plus pour adapter leurs comptes.

Les comptes établis selon les nouvelles normes comprennent les parties suivantes: bilan, annexe, tableau des flux de trésorerie (pour les grandes organisations), tableau de variation du capital et rapport de performance. Ce dernier élément fournit des informations de nature qualitative (efficience et efficacité), qui viennent compléter la partie quantitative. Désormais, il faut publier en outre les chiffres relatifs aux prestations reçues gratuitement ou à prix préférentiel telles que le bénévolat, les dons en nature, les rabais spéciaux, les prestations fournies à titre gracieux en cas d'achat de marchandises, etc. La vérification du rapport de performance ne fait pas partie des compétences ordinaires de l'organe de révision, mais incombe uniquement à la ZEWO. 


\section{Frais de gestion}

De plus en plus de donateurs, de bailleurs de fonds institutionnels, mais aussi de médias réclament des chiffres sur les frais de gestion des institutions d'utilité publique, chiffres qu'ils estiment révélateurs de l'efficacité et de la vigilance des organisations, notamment au niveau de l'affectation des dons. Il s'agit là d'une question complexe. En effet, il n'est pas possible de déterminer sur la base d'une seule donnée si les dons ont été utilisés à bon escient. De plus, les comparaisons de chiffres entre différentes institutions posent problème.

Si les nouvelles recommandations relatives à la présentation des comptes (RPC 21) indiquent les chiffres qui doivent être publiés, elles ne donnent aucune définition précise des frais de gestion. Ainsi, les institutions continuent de décider elles-mêmes quelles données elles souhaitent intégrer à ce poste. La plupart d'entre elles y font figurer les frais administratifs généraux et les frais liés aux collectes, sans les coûts occasionnés par le suivi des projets. Ainsi, le montant des frais de gestion généraux varie en fonction des coûts que les institutions attribuent aux projets.

La ZEWO a lancé mi-juin 2004 un projet visant à clarifier le concept de frais de gestion et à en donner une définition. Les premières évaluations sont attendues fin 2005 .

\section{Degré de notoriété du label ZEWO}

Selon une étude récente de Publitest SA, le degré de notoriété spontanée du label de qualité ZEWO est resté stable depuis 2001 auprès de l'ensemble de la population, avec un chiffre de $21 \%$. Il a en revanche nettement augmenté auprès des personnes qui font des dons réguliers (43\% en 2004 contre $32 \%$ en 2001). La ZEWO jouit d'une plus grande notoriété en Suisse alémanique qu'en Suisse romande. Quant au label de qualité ZEWO, il est plus connu auprès des femmes de plus de 30 ans à revenu élevé (plus de 6000 francs par mois) et des personnes ayant suivi une formation supérieure. $63 \%$ des personnes interrogées jugent le label de qualité crédible ou très crédible. Les donateurs réguliers ou occasionnels lui accordent $73 \%$ de crédibilité.

\section{Conclusion}

La promotion de l'intégrité et de la transparence en matière de dons répond sans conteste à un besoin du grand public. La ZEWO y apporte une contribution importante, notamment en exigeant des organisations qui souhaitent obtenir son label de qualité qu'elles mettent en place des structures de contrôle efficaces, qu'elles fassent appel à un organe de révision pour leur contrôle de gestion et qu'elles publient des rapports et des comptes annuels pour informer leurs groupes d'interlocuteurs. Ces questions ne cessent de gagner en importance, en particulier dans le cadre de la corporate governance. La ZEWO ne peut certes pas garantir la sécurité absolue dans l'utilisation des dons par les institutions d'utilité publique, mais il lui incombe de veiller à l'adoption de mesures de contrôle appropriées. 
Dans cette perspective, l'objectif de la ZEWO est d'intensifier le dialogue avec ces institutions sur une base consolidée et de promouvoir son label de qualité, afin que celui-ci devienne auprès du grand public un gage d'efficacité et de fiabilité. Par ailleurs, la ZEWO souhaite renforcer à moyen terme sa présence en Suisse romande.

\section{Statistique des dons}

Les statistiques de la ZEWO pour 2001 et 2002 englobent les dons faits pendant cette période aux quelque 300 institutions ayant reçu le label de qualité. Contrairement au baromètre des dons de la GfS (Société suisse de recherches sociales pratiques), qui est basé sur un sondage effectué auprès des donateurs, les évaluations de la ZEWO se fondent sur les données fournies par les œuvres d'entraide (questionnaire écrit et comptes annuels).

Les recettes totales des institutions titulaires du label de qualité ZEWO s'élevaient à 1,7 milliard de francs en 2002 , soit un montant comparable à celui enregistré l'année précédente. Les institutions actives dans la coopération au développement génèrent quelque $40 \%$ de ces recettes.

Les principales sources de revenu des institutions collectant des dons habilitées à utiliser le label ZEWO sont les dons privés (610 millions de francs, soit $36 \%$ des revenus) et les contributions publiques (580 millions de francs, soit $34 \%$ des revenus). En comparaison avec l'année 2001, durant laquelle les chiffres ont atteint des niveaux exceptionnels, les dons privés ont diminué de $13 \%$. Ils sont répartis entre legs, dons individuels, dons d'institutions (Eglises, fondations, associations), cotisations de membres, etc. On remarque une baisse des cotisations de membres, des dons d'institutions et des dons individuels, lesquels enregistrent le recul le plus net (100 millions de francs). Les legs, en revanche, ont augmenté de 15 millions de francs. La même tendance positive est à observer pour les contributions publiques (hausse de 40 millions de francs environ) et les prestations propres des institutions (hausse de 10 millions de francs). 
Graphique 1: Répartition du total des recettes (CHF 1,7 milliard) des organisations certifiées ZEWO ( $n=280)$ en 2002

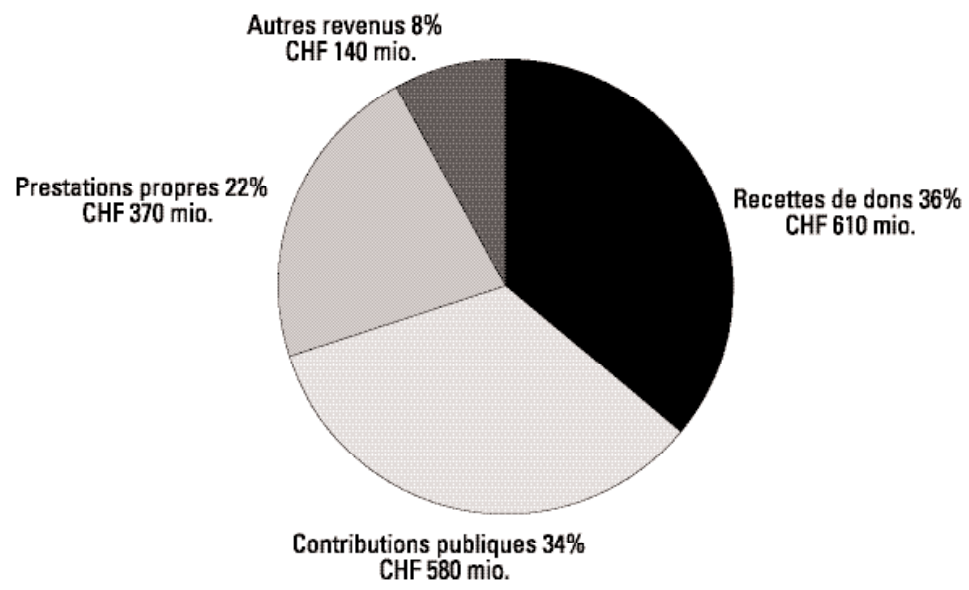

Graphique 2: Répartition du total des recettes (CHF 1,7 milliard) des organisations certifiées ZEWO ( $n=280)$ en 2001

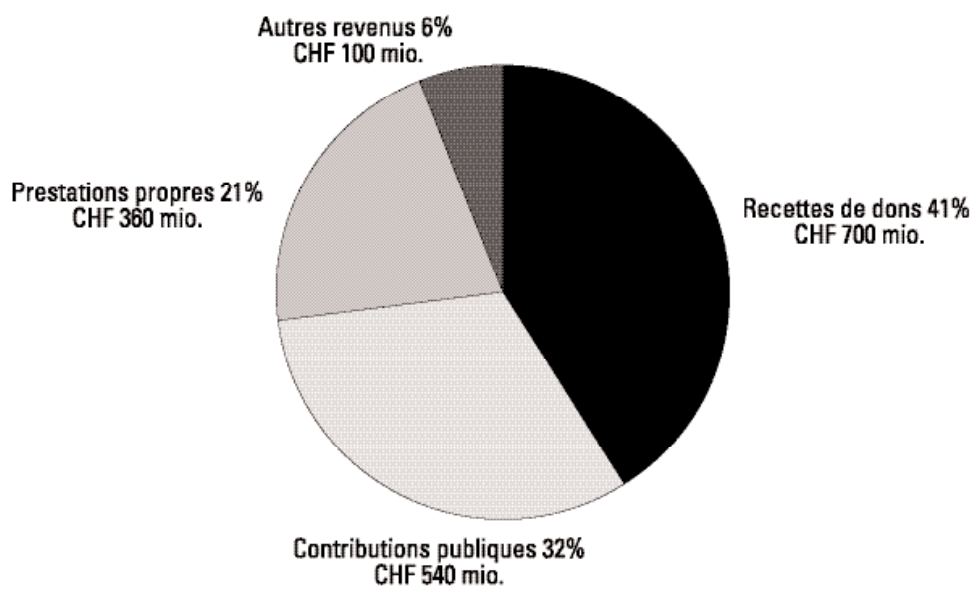


Graphique 3: Recettes de dons (CHF 610 millions) des organisations certifiées ZEWO ( $n=280)$ en 2002

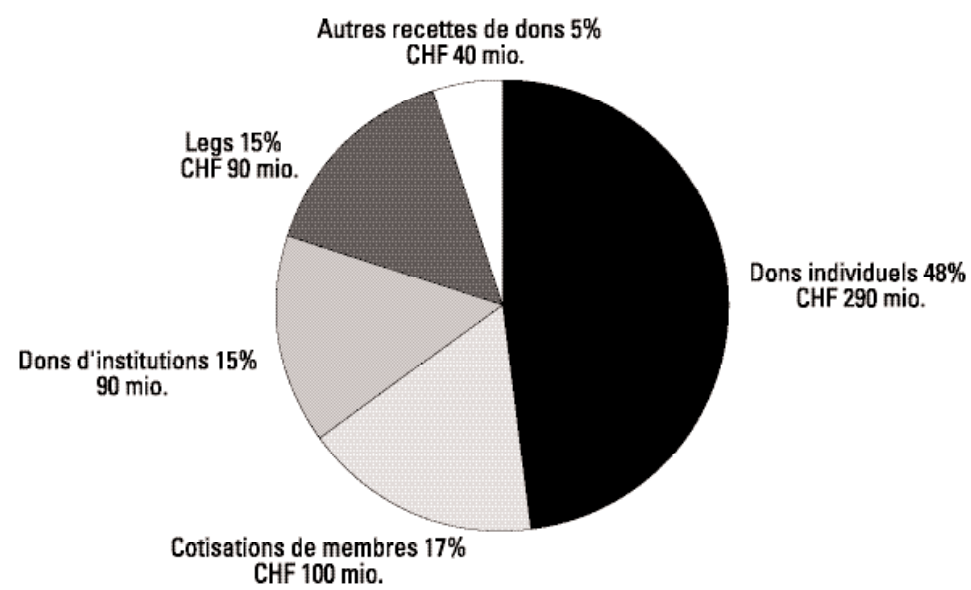

Graphique 4: Recettes de dons (CHF 700 millions) des organisations certifiées ZEWO ( $n=280)$ en 2001

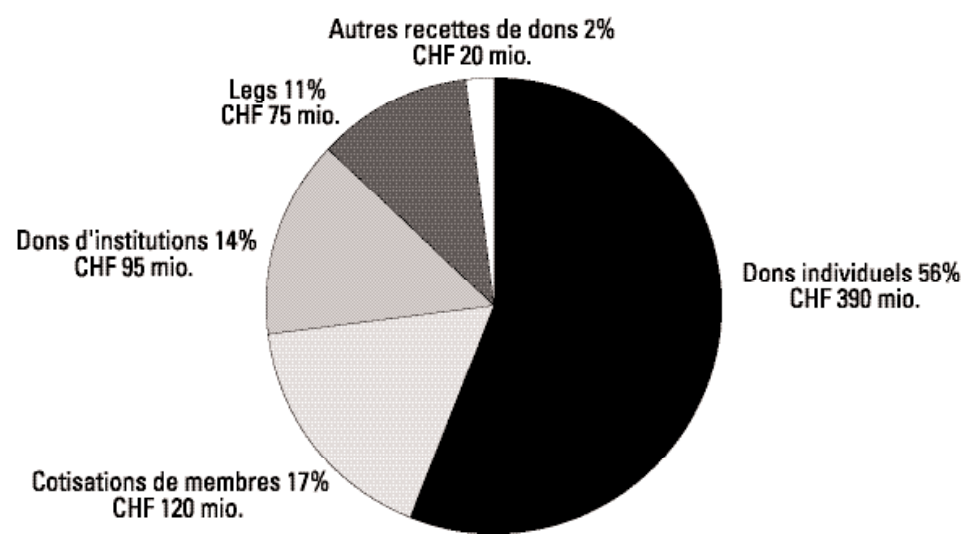

